\title{
Indicaciones no tradicionales de amigdalectomía
}

\author{
Nontraditional indications for tonsillectomy \\ Cristián Aedo B', Daniel Muñoz S².
}

\begin{abstract}
RESUMEN
La amigdalectomía es la intervención quirúrgica más frecuente en otorrinolaringología y sus indicaciones son ampliamente conocidas en la especialidad. El avance en el conocimiento de sus funciones inmunológicas ha determinado su uso en el tratamiento de otras enfermedades tales como la nefropatía por $\lg A$, síndromes neuropsiquiátricos, el síndrome de fiebre recurrente, algunas entidades dermatológicas, entre otras. Pretendemos así, revisar la literatura para evaluar la evidencia disponible que sustente lo que denominamos indicaciones no tradicionales. Al parecer, la evidencia a favor del uso de amigdalectomía en el tratamiento de algunas de estas enfermedades es sólido, mientras que para otras aún no supera la suposición teórica, pues sólo se cuenta con casos aislados. Consideramos necesario estudios más extensos, con mayor calidad metodológica para definir mejor la indicación de amigdalectomía. Esto, más la evaluación multidisciplinaria de cada caso nos debiera conducir a la mejor decisión.
\end{abstract}

Palabras clave: Amigdalectomía, nefropatía por IgA, indicación quirúrgica, fiebre recurrente.

\begin{abstract}
Tonsillectomy is the most common surgical procedure in otorhinolaryngology and their indications are well known in the field. The advance in knowledge of their immune function has determined its use in the treatment of other diseases such as $\lg A$ nephropathy, neuropsychiatric syndromes, periodic fever syndrome, some dermatologic entities, and others. We review the literature to evaluate the available evidence that supports what we call nontraditional indications. Apparently, the evidence for the use of tonsillectomy in the treatment of some diseases is solid, while others still not overcome the theoretical assumption, since there are only isolated cases. Larger studies are needed to consider, with higher methodological quality, to better define the indication for tonsillectomy. This and the multidisciplinary assessment of each case should lead us to the best decision.
\end{abstract}

Key words: Tonsillectomy, IgA nephropathy, surgical indication, periodic fever.

1 Médico Otorrinolaringólogo. Hospital Clínico San Borja Arriarán.

2 Médico Cirujano. Universidad de Chile. 


\section{INTRODUCCIÓN}

Las amígdalas palatinas son masas linfoides ovoides que forman parte del anillo de Waldeyer, y están localizadas lateralmente en la orofaringe. Su rol es participar en el sistema inmunológico a través de inmunidad local, mediante la producción de inmunoglobulinas, principalmente IgA y también mediante la presentación de antígenos ${ }^{1}$. Dada estas funciones y su ubicación anatómica, las amígdalas son susceptibles de producir obstrucción por hiperplasia y de sufrir procesos infecciosos-inflamatorios a repetición, que han determinado históricamente su indicación de extracción. La amigdalectomía es la intervención quirúrgica más frecuente en otorrinolaringología sobre todo en la edad pediátrica y generalmente acompañada de adenoidectomía. Su técnica quirúrgica ha evolucionado notablemente con los años y actualmente la mayoría de ellas tienden a facilitar la resección y disminuir el sangrado, consiguiendo así un menor tiempo quirúrgico y menor comorbilidad².

La Academia Americana de Otorrinolaringología publicó en 1995 en el Clinical Indicators Compendium, las guías clínicas e indicaciones de amigdalectomía que incluía 30 más infecciones por año, hipertrofia que produzca comorbilidad, absceso periamigdalino, halitosis debido a amigdalitis crónica, amigdalitis crónica recurrente con portación de Streptococcus, hipertrofía unilateral 0 sospecha de neoplasia ${ }^{3}$.

Con anterioridad, los estudios de Paradise y cols en 1984, que buscaban evaluar la eficacia de la amigadalectomía en pacientes con faringoamigdalitis recurrente, determinaron algunos criterios para la indicación de amigdalectomía por dicha causa. Estos son: 70 más episodios en un año, 50 más por año en los últimos 2 años 030 más por año en los últimos 3 años ${ }^{4}$. Dichos criterios han sido también adoptados por muchos médicos, como parte de las indicaciones tradicionales de amigdalectomía. En la actualidad las indicaciones se clasifican en indicaciones absolutas y relativas (Tabla 1).

Sin embargo lo anterior, se han descrito algunas entidades en cuyo tratamiento se incluye el uso de amigdalectomía. Este hecho, se basa en que existirían algunas enfermedades que en su patogenia jugarían un rol relevante las funciones inmunológicas amigdalinas como la producción de IgA por ejemplo en la nefropatía por depósito de inmunoglobulina $A(\lg A)^{5}$, y por otro lado enfermedades que estarían relacionadas con trastornos autoinmunes producto de autoanticuerpos en infecciones estreptocócicas ${ }^{6} 0$ en portadores del estreptococo a nivel amigdalino, y que estaría relacionado con trastornos neuropsiquiátricos, en el síndrome de fiebre recurrente ${ }^{7}$ y en la psoriasis.

Con nuestra revisión pretendemos establecer si existen evidencias científicas que avalen la amigdalectomía en enfermedades como: nefropatía por IgA, PANDAS, síndrome de fiebre recurrente y psoriasis, entre otras, de manera de aplicarlas a nuestra práctica interdisciplinaria habitual cuando corresponda.

\section{NEFROPATÍA POR IGA Y AMIGDALECTOMÍA}

La nefropatía por IgA o enfermedad de Berger, es la forma más común de enfermedad glomerular en el mundo, dando cuenta de $25 \%-50 \%$ de las glomerulonefritis primarias ${ }^{8}$. Se caracteriza por la presencia de depósitos glomerulares de IgA y proliferación mesangial. Clínicamente se presenta

Tabla 1. Criterios actuales para la indicación de amigdalectomía.

\begin{tabular}{|c|c|}
\hline Criterios mayores & Criterios menores \\
\hline $\begin{array}{l}\text { - } \quad \text { Cáncer de amígdala } \\
\text { - Obstrucción grave de la vía aérea a con } \\
\text { hipoxia o hipercapnia }\end{array}$ & $\begin{array}{ll}\text { - } & \text { Amigdalitis a repetición } \\
\text { (según los criterios de Paradise y cols) } \\
\text { - } & \text { Absceso periamigdalino } \\
\text { - } & \text { Halitosis de origen amigdalino }\end{array}$ \\
\hline
\end{tabular}


con hematuria macroscópica simultáneamente con infecciones respiratorias altas en pacientes jóvenes o con alteraciones del sedimento urinario y proteinuria en pacientes asintomáticos. Más de la mitad de los pacientes puede, con el tiempo, progresar a insuficiencia renal crónica9.

La relación patogénica con las amígdalas se fundamenta en que éstas son estructuras que producen anticuerpos, entre ellos IgA y que se ha visto que la amigdalectomía disminuye los niveles séricos y la secreción salival de IgA ${ }^{10}$. Por otro lado, se ha demostrado que los depósitos de IgA en el mesangio glomerular tienen en parte importante un origen amigdalino ${ }^{11}$ (Figura 1). Se han descrito varias estrategias de tratamiento para la enfermedad de Berger, que incluyen la amigdalectomía, el uso de corticoides, inmunosupresores, antihipertensivos, anticoagulantes, entre otros ${ }^{12}$.

La amigdalectomía puede mejorar el deterioro de la función renal, mantener este deterioro estable y puede tener un efecto favorable en términos de sobrevida a largo plazo en algunos pacientes con nefropatía por IgA, principalmente porque produciría disminución de la secreción de IgA y consecutivamente producir disminución de la proteinuria y hematuria ${ }^{13}$. Actualmente, gran parte de los estudios que consideran la amigdalectomía exclusiva como tratamiento son de tipo retrospectivo y existe poca información sobre complicaciones de esta intervención en este tipo de pacientes. El único estudio prospectivo que destaca es el de Akagi y cols $^{14}$, que trataron 24 pacientes con amigdalectomía y los observó por un periodo de 2 años, logrando una remisión de la proteinuria en $50 \%$ de los casos. Por otro lado, Chen y cols $^{15}$ en un estudio de casos y controles, encontró una diferencia significativa en la remisión clínica de la enfermedad entre los que recibieron amigdalectomía y aquellos que no (Tabla 2).

También se ha usado terapia combinada de amigdalectomía con corticoides sistémicos en pacientes con nefropatía por IgA, lo que al parecer también tendría repercusión sobre la mejoría de la función renal, pero sin definición sobre la sobrevida (Tabla 3). Destacan en este sentido los trabajos de Hotta y cols, quienes han visto retrospectiva y prospectivamente cómo la terapia combinada repercute positivamente en mejorar la función renal, sobre todo en etapas iniciales del daño renal $^{16-18}$.

Sato y $\operatorname{cols}^{19}$, proponen en base a un estudio retrospectivo en 70 pacientes con nefropatía por $\lg$ A, que la terapia combinada sería eficaz también en disminuir la progresión hacia la insuficiencia

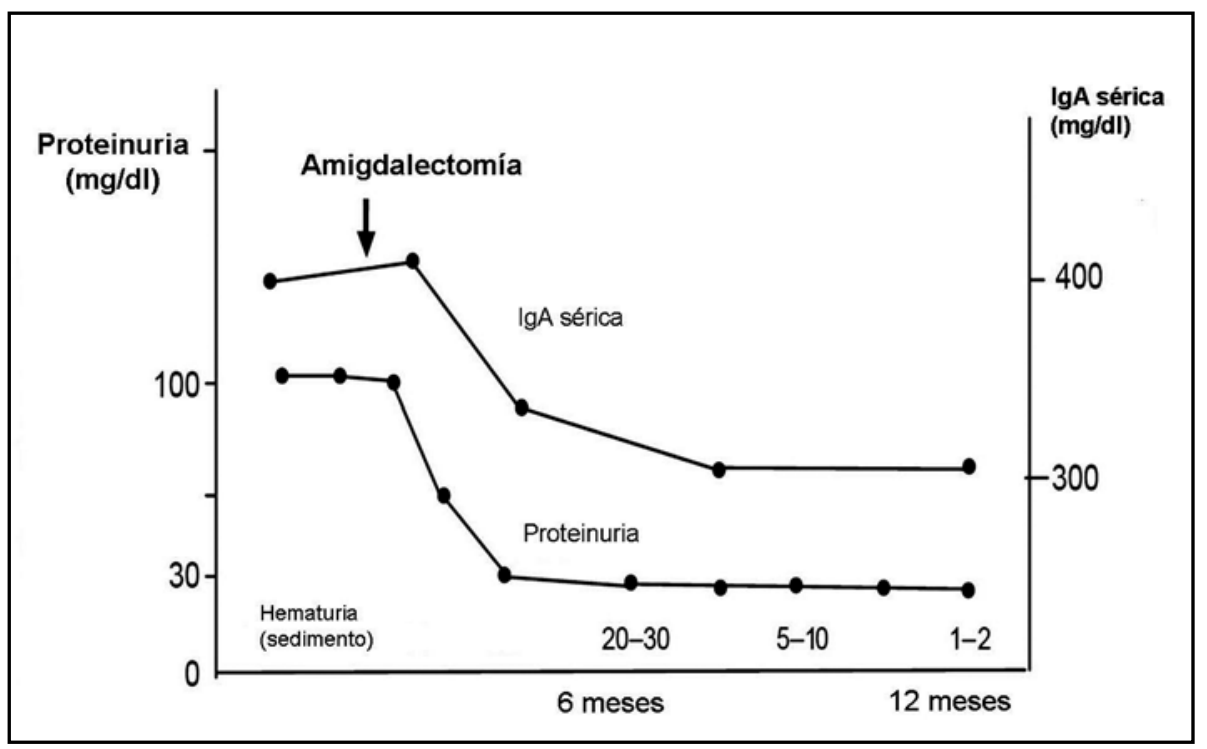

Figura 1. Evolución de un paciente con nefropatía por IgA, luego de una amigdalectomía. Se observa un descenso progresivo de la IgA sérica, proteinuria y hematuria posterior a la intervención. (Extraído de Yambe H et al. Acta Otolaryngol Suppl. 2004 Dec;(555):54-7.) 
Tabla 2. Estudios clínicos sobre el uso de amigdalectomía en el tratamiento de la nefropatía por IgA (Am =amigdalectomía)

\begin{tabular}{|c|c|c|c|c|}
\hline Autor & Diseño & Pacientes & Seguimiento & Resultados \\
\hline Tomioka $(1996)^{21}$ & Serie de casos & 15 & 1 año & $86 \%$ mejoría clínica \\
\hline Sanai et al. $(1996)^{22}$ & Casos y controles & $\begin{array}{l}8(\mathrm{Am}) \\
7 \text { controles }\end{array}$ & 1 año & $62 \%$ mejoría clínica \\
\hline Rasche at al. $(1999)^{23}$ & $\begin{array}{l}\text { Retrospectivo casos } \\
\text { y controles }\end{array}$ & $\begin{array}{l}16(\mathrm{Am}) \\
39 \text { controles }\end{array}$ & 26 años & $\begin{array}{l}\text { Sin diferencia significativa } \\
\text { en sobrevida y progresión a } \\
\text { cronicidad }\end{array}$ \\
\hline Xie et al. (2003)21 & $\begin{array}{l}\text { Retrospectivo casos y } \\
\text { controles }\end{array}$ & $\begin{array}{l}48(\mathrm{Am}) \\
70 \text { controles }\end{array}$ & $192,9+/-74,8$ meses & $\begin{array}{l}\text { A sobrevida y } \\
\text { progresión a cronicidad }\end{array}$ \\
\hline Agaki et al. (2004)14 & $\begin{array}{l}\text { Retrospectivo casos y } \\
\text { controles }\end{array}$ & $\begin{array}{l}41(\mathrm{Am}) \\
30 \text { controles }\end{array}$ & 10 años & $\begin{array}{l}\text { Am } 24,4 \% \text { remisión clínica } \\
\text { y } 95,1 \% \text { sobrevida versus } 13,3 \% \\
\text { y } 73,3 \% \text { respectivamente }\end{array}$ \\
\hline Yumabe et al. (2004)10 & Retrospectivo & $\begin{array}{l}36(\mathrm{Am}) \\
26 \text { controles }\end{array}$ & 22 años & proteinuria y hematuria \\
\hline Nishi et al. (2004)24 & $\begin{array}{l}\text { Retrospectivo casos y } \\
\text { controles }\end{array}$ & $\begin{array}{l}46(\mathrm{Am}) \\
74 \text { controles }\end{array}$ & $197+/-29,3$ meses & Am sobrevida \\
\hline Chen et al. (2007)15 & $\begin{array}{l}\text { Retrospectivo casos y } \\
\text { controles }\end{array}$ & $\begin{array}{l}54(\mathrm{Am}) \\
58 \text { controles }\end{array}$ & 130 meses & $\begin{array}{l}\text { Am } 46,3 \% \text { remisión clínica versus } \\
27,6 \% \text { en controles }\end{array}$ \\
\hline
\end{tabular}

Tabla 3. Evidencia en la literatura sobre uso de terapia combinada de amigdalectomía y corticoides en nefropatía IgA (Am=amigdalectomía; $C=$ corticoides)

\begin{tabular}{|c|c|c|c|c|}
\hline Autor & Diseño & Pacientes & Seguimiento & Resultado \\
\hline Hotta et al. (2001) & retrospectivo & 329 & $82,3+/-38,2$ meses & 48\% remisión clínica \\
\hline Hotta et al. (2002) & prospectivo & 35 & 77,1 meses promedio & Hasta $100 \%$ en etapas iniciales \\
\hline Sato et al. (2003) & retrospectivo & $\begin{array}{l}30(\mathrm{Am}+\mathrm{C}) \\
25(\mathrm{C}) \\
15 \text { (soporte) }\end{array}$ & & $\begin{array}{l}\mathrm{Am}+\mathrm{C} \text { mejor en_proteinuria } \\
\text { y evitar progresión a cronicidad }\end{array}$ \\
\hline Hotta (2004) & prospectivo & 372 & 3 años & 59\% remisión clínica \\
\hline Kawasaki et al. (2006) & Prospectivo randomizado & $\begin{array}{l}16(\mathrm{Am}+\mathrm{C}) \\
16 \text { (otros) }\end{array}$ & 2 años & $\begin{array}{l}\text { Am+C mejor en_proteinuria } \\
\text { y severidad histológica }\end{array}$ \\
\hline Miyazaki et al. (2007) & Prospectivo multicéntrico & $\begin{array}{l}75(\mathrm{Am}+\mathrm{C}) \\
16(\mathrm{C})\end{array}$ & 5 años & $\mathrm{Am}+\mathrm{C}$ mejor en_proteinuria \\
\hline Suwabe et al. (2007) & prospectivo & 72 pacientes & $20,3+/-9,7$ meses & 93\% remisión clínica \\
\hline Komatsu et al. (2008) & Prospectivo controlado & $\begin{array}{l}35(A m+C) \\
20(C)\end{array}$ & $54+/-21,2$ meses & $\begin{array}{l}\text { Am+C mejor en } \\
\text { proteinuria y hematuria }\end{array}$ \\
\hline
\end{tabular}

renal crónica. Según Kawasaki y cols ${ }^{20}$, la terapia combinada sería mejor en disminuir la magnitud de la proteinuria y evitaría el progreso del daño histológico y, por ende, de la enfermedad. No existen estudios que comparen la amigdalectomía sola versus terapia combinada, pues la mayoría de los trabajos se han realizado con controles.

En resumen, existe evidencia científica que avala principalmente la amigdalectomía combinada con corticoterapia en el manejo de la nefropatía por 
IgA, la cual sería recomendable en pacientes con deterioro de la función renal leve o moderada, ya que en pacientes con daño renal severo no cambiaría el pronóstico, debido a su irreversibilidad.

El manejo de este tipo de pacientes requiere de un equipo especializado, donde al trabajo de nefrólogos se agrega cada vez con mayor fuerza la intervención de los otorrinolaringólogos.

\section{PANDAS Y AMIGDALECTOMÍA}

El síndrome PANDAS (Pediatric Autoinmune Neuropsychiatric Disorders Associated with Streptococcus) es una entidad rara que se describió por primera vez en $1998^{6}$. Describe la presencia de trastorno obsesivo-compulsivo (TOC) o la presencia de tics con un curso episódico y una relación temporal con infecciones causadas por Estreptococo beta hemolítico del grupo A $(\mathrm{SBHGA})^{25}$. Es frecuente también encontrar comorbilidad del tipo labilidad emocional, ansiedad, déficit atencional e hiperactividad ${ }^{26}$. Los pacientes con esta afección presentan una exacerbación dramática de los síntomas neuropsiquiátricos con las infecciones amigdalinas causadas por SBHGA 27 (Tabla 4).

La fisiopatología del síndrome PANDAS se postula que es semejante a la del corea de Sydenham y otros desórdenes neuropsiquiátricos de tipo autoinmune. Se cree que los autoanticuerpos generados por una reacción inmunitaria cruzada con SBHGA, actuarían en los ganglios basales dando lugar a las alteraciones del movimiento y del comportamiento antes mencionadas ${ }^{28}$.

La manera óptima de tratar estos pacientes aún no ha sido establecida. La profilaxis con penicilina no ha probado ser efectiva y otros tratamientos están aún en etapa experimental ${ }^{26}$. Existe alguna evidencia en la literatura que apoya el rol que tendría la amigdalectomía en mejorar esta condición.

Orvidas y cols ${ }^{29}$ reportaron el caso de 2 pacientes para los cuales la amigdalectomía resultó efectiva, no presentando exacerbaciones de su sintomatología neuropsiquiátrica durante el tiempo de seguimiento posoperatorio.

Heubie y cols ${ }^{30}$ reportaron 2 casos de pacientes con criterios de PANDAS y amigdalitis recurrente que fueron tratados exitosamente con amigdalectomía, en cuanto a su sintomatología neuropsiquiátrica. Batuecas y cols ${ }^{31}$, publicaron un caso de un paciente con síndrome PANDAS sometido a amigdalectomía que mejoró de su sintomatología tras la intervención. Del mismo modo, Lynch y cols ${ }^{32}$, publicaron en 2006 un caso de un niño con TOC y alteraciones del movimiento asociadas a infecciones faríngeas recurrentes pos SBHGA y que mejora clínicamente luego de ser sometido a amigdalectomía.

En resumen, existe evidencia científica que marca una tendencia para indicar la amigdalectomía en pacientes que presenten PANDAS. Por lo tanto, debiéramos estar atentos a la posibilidad de encontrar esta enfermedad en niños que se presentan con amigdalitis recurrente $y$ comorbilidad neuropsiquiátrica, por lo que la anamnesis debe incluir preguntas relacionadas a buscar relación entre exacerbación de síntomas e infecciones estreptocócicas.

\section{SÍNDROME PFAPA Y AMIGDALECTOMÍA}

El síndrome PFAPA (periodic fever, aphtous stomatitis, pharyngitis and adenitis) es una entidad

Tabla 4. Criterios diagnósticos del síndrome PANDAS

1. Presentación de tics 0 TOC ( 0 ambos)

2. Inicio en la infancia (entre los 3 años y la pubertad).

3. Presentación episódica (inicio brusco de síntomas, con remisión posterior y nueva reaparición brusca del cuadro).

4. Asociación de la exacerbación con infección por SBHGA (determinado por cultivo faríngeo o elevación de anticuerpos)

5. Asociación con signos neurológicos anormales (movimientos anormales, hiperactividad, entre otros). 
relativamente recién descrita por Marshall en 1987 como un síndrome de fiebre recurrente en presencia de aftas bucales, faringitis y adenitis cervical ${ }^{7}$. Se cree que PFAPA tiene un curso prolongado con tendencia a la mejoría espontánea con el tiempo, pero dada su naturaleza recurrente puede requerir una evaluación mayor ${ }^{33}$.

Clínicamente se manifiesta con fiebre casi en la totalidad de los casos, las adenopatías cervicales en $85 \%-88 \%$ de los pacientes, seguida de faringitis en $72 \%$ y de estomatitis con aftas bucales en $70 \%$ de los sujetos. La mayoría de los brotes comienzan antes de los 5 años. Se puede asociar a cefalea, náuseas, vómitos y dolor abdominal ${ }^{34}$.

En la actualidad, la etiología de este síndrome permanece incierta. Algunos autores postulan la naturaleza inflamatoria, en base a la respuesta obtenida en algunos pacientes tratados con corticoides y la detección de elevación de algunos parámetros inflamatorios como citoquinas ${ }^{35}$.

Los intentos de tratamiento de este síndrome provienen de series de casos, en ausencia de estudios controlados. Consiste predominantemente, en el uso de corticoides sistémicos, con una mejoría de hasta $76 \%$, pero con mucha recurrencia ${ }^{36}$. Se ha usado también cimetidina para prevenir las recurrencias y disminuir la severidad, pero con éxito limitado ${ }^{37}$.

Se han reportado en la literaturas varias series de casos que describen el uso de la amigdalectomía en pacientes con PFAPA, logrando mejoría sintomática variable según la serie. Los reportes más recientes al respecto han mostrado efectividad considerable de la amigdalectomía en detener la enfermedad. Este hecho ha hecho suponer que la enfermedad estaría originada por algún proceso de tipo inmunológico, originado en el tejido amigdalino (Tabla 5).

Tasher y cols ${ }^{38}$, observaron durante 5 años un grupo de pacientes con síndrome de PFAPA, de los cuales 6 fueron amigdalectomizados, obteniendo $100 \%$ de remisión sintomática finalizado el seguimiento. Licameli et a ${ }^{40}$ revisaron retrospectivamente una serie de 27 pacientes con síndrome PFAPA amigdalectomizados obteniendo mejoría clínica en $26(96,2 \%)$ de ellos. Wong y cols ${ }^{41}$ presentaron una serie de 9 casos, de los cuales 8 mejoraron significativamente a 3 meses de seguimiento.

Renko y cols $^{39}$ han realizado el único estudio prospectivo, randomizado y controlado sobre la utilidad de amigdalectomía en PFAPA. Ellos obtuvieron respuesta clínica total a la intervención en 14 pacientes una vez finalizado el seguimiento.

\section{PATOLOGÍA DERMATOLÓGICA Y AMIGDALECTOMÍA}

En dermatología es bien conocido que las infecciones estreptocócicas de las amígdalas afectan el curso de ciertas enfermedades dermatológicas tales como la pustulosis palmo-plantar (PPP) y la psoriasis (gutatta y crónica en placas), enfermedades que comparten una base patogénica de tipo inmunológica.

Tabla 5. Efectividad de amigdalectomía en PFAPA

\begin{tabular}{|lccc|}
\hline Autor & No $^{\circ}$ pacientes & Tratamientos previos & Éxito post-amigdalectomía (\%) \\
\hline Galanakis y cols $(2002)^{42}$ & 15 & No & 100 \\
Berlucchi y cols $(2003)^{43}$ & 5 & Corticoides & 100 \\
Thomas y cols $(1999)^{35}$ & 11 & Corticoides & 64 \\
Dahn y cols $(2000)^{44}$ & 4 & No & 100 \\
Parikh y cols $(2003)^{45}$ & 2 & Cimetidina & 0 \\
Tasher y cols $(2006)^{38}$ & 6 & No & 100 \\
Wong y cols $(2008)^{41}$ & 9 & No & 88,8 \\
Licameli y cols $(2008)^{40}$ & 27 & No & 96,2 \\
Renko y cols $(2007)^{39}$ & 14 & No & 100 \\
\hline
\end{tabular}


La PPP es una dermatosis crónica recurrente caracterizada por la presencia de pústulas intraepidérmicas y eritema palmo-plantar. Tradicionalmente se ha sugerido que la acción de citoquinas proinflamatorias juegan un rol central en la génesis de esta enfermedad. La amigdalectomía produce una reducción de los niveles plasmáticos de IL-8 e IL-6 en pacientes con PPP, sugiriendo que dichas citoquinas están relacionadas con la producción de la enfermedad a partir de infecciones amigdalinas ${ }^{46}$.

En el caso de la psoriasis, es sabido que las infecciones estreptocócicas producen activación de células T epidérmicas, lo que finalmente inducirá la manifestación de la enfermedad. En este caso, los principales actores son los superantígenos estreptocócicos, que pueden indirectamente inducir al llamado antígeno linfocítico cutáneo facilitando así, la migración de los linfocitos $T$ desde los capilares dérmicos hacia la epidermis ${ }^{47}$.

Si bien no existe evidencia concluyente sobre el beneficio de la amigdalectomía en psoriasis, es frecuente en la literatura dermatológica la recomendación de su uso, sobre todo en aquellos en que los tratamientos convencionales no dan resultado.

Entre las observaciones más importantes en este ámbito, destacan: McMillin y cols48 quienes reportaron 2 casos de niños con psoriasis que fueron amigdalectomizados y quedaron completamente libres de enfermedad durante el periodo de observación de 16 meses. Nyfors ${ }^{49}$ y cols, realizó un estudio retrospectivo con 74 pacientes con psoriasis adultos y niños, que fueron amigdalectomizados y seguidos por 5 años, encontrando una buena respuesta a tratamiento, con $71 \%$ de remisión de la enfermedad. Del mismo modo, Hone ${ }^{50}$ y cols revisaron los casos de 13 pacientes adultos y niños que luego de su amigdalectomía mostraron $76 \%$ de buena respuesta clínica luego de 26 meses de observación.

Es de esperar que en un futuro próximo, la relación fisiopatológica entre el tejido amigdalino y la producción de PPP y psoriasis sea dilucidada con mayor claridad, así como también se demuestre el beneficio de la amigdalectomía en el manejo de estos pacientes, adquiriendo una importancia mucho mayor a la actual.

\section{OTRAS PATOLOGÍAS}

Se ha mencionado la utilidad de la amigdalectomía en el tratamiento de una serie de patologías tales como la erradicación de Helycobacter pylor ${ }^{51}$ (por portación en tejido amigdalino), invaginación intestinal $^{52}$, uveítis postestreptocócica recurrente ${ }^{53}$ y protección contra la aparición enfermedades linfoproliferativas en transplantados hepáticos ${ }^{54,55}$. Dichas afirmaciones se han basado sólo en observaciones de casos 0 asociaciones epidemiológicas (como es el caso de Helicobacter pylori e invaginación intestinal) por lo que su verdadero rol es aún incierto, así como también el mecanismo patogénico relacionado, lo que abre nuevas líneas de investigación en esta materia.

\section{CONCLUSIONES}

A pesar que la amigdalectomía se viene realizando hace más de 100 años, es hasta hace unos pocos años que se ha comenzado a estudiar las funciones inmunológicas y/o microbiológicas inherentes a la presencia 0 ausencia de ellas en los pacientes de diversos grupos etarios. Es indiscutible los beneficios en amigdalitis crónica como en síndromes de apnea-hipopneas obstructiva del sueño, sin embargo, los estudios realizados y los argumentos científicos en las diversas indicaciones no tradicionales de amigdalectomía analizadas en esta revisión son muy discutibles.

Actualmente es conocida la etiopatogenia y el rol beneficioso de la amigdalectomia en pacientes con PANDA, Nefropatía por IgA y síndrome PFAPA, acreditado por la serie de estudios clínicos revisados.

En la serie de patologías de origen dermatológico, existen solo inclinaciones de tipo teórico para su indicación, sin embargo, aún no han podido demostrase fehacientemente.

Consideramos necesario estudios más extensos, con mayor calidad metodológica para definir mejor la indicación de amigdalectomía. Esto, más la evaluación multidisciplinaría de cada caso nos debiera conducir a la mejor decisión.

Es evidente de todas maneras que debemos tomar una actitud crítica y analítica frente a la 
aparición de nuevas tendencias clínicas que alcanzan nuestra especialidad, debido a la escasa evidencia que sustenta algunas de estas «modas»; pero es indispensable estar dispuesto al trabajo y al estudio multidisciplinario.

\section{BIBLIOGRAFÍA}

1. Ishikawa T, Wicher K, Arbesman CE. Distribution of immunoglobulins in palatine and pharyngeal tonsils. Int Arch Allergy Appl Immunol 1972; 43(6): 801-12.

2. Mattila Ps, Tahkokallio 0, Tarkkanen J, Pitkäniemi J, Karvonen M, Tuomilehto J. Causes of tonsillar disease and frequency of tonsillectomy operations. Arch Otolaryngol Head Neck Surg 2001; 127(1): 37-44.

3. American Academy of Otolaryngology-Head and Neck Surgery. 1995 Clinical indicators compendium, Alexandria, Virginia, 1995, American Academy of Otolaryngology-Head and Neck Surgery.

4. Paradise Jl, Bluestone CD, Bachman RZ, Colborn DK, Bernard BS, Taylor FH et al. Efficacy of tonsillectomy for recurrent throat infection in severely affected children. Results of parallel randomized and nonrandomized clinical trials. N Engl J Med 1984; 310(11): 674-83.

5. Coppo R. Pediatric IgA nephropathy: clinical and therapeutic perspectives. Semin Nephrol 2008; 28(1): 18-26.

6. Swedo Se, Leonard HL, Garvey M, et al. Pediatric autoimmune neuropsychiatric disorders associated with streptococcal infections: clinical description of the first 50 cases. Am J Psychiatry 1998; 155: 264-271.

7. Marshall GS, Edwards KM, Lawton AR. PFAPA syndrome. Pediatr Infect Dis J 1989; 8: 658659.

8. ROStOker G. Therapy of IgA nephropathy. BioDrugs 1998; 9(4): 279-301.

9. Donadio JV, Grande JP. IgA nephropathy. N Engl J Med 2002; 347(10): 738-48.

10. Yamabe $H$, Sugawara T, Nakamura M, Shimada $M$. Involvement of tonsils in IgA nephropathy. Acta Otolaryngol Supp/ 2004; (555): 54-7.

11. XIe Y, Chen X, Nishi S, Narita I, Gejyo F. Relationship between tonsils and $\lg A$ nephropathy as well as indications of tonsillectomy. Kidney Int 2004; 65(4): 135-44.

12. Kodama S, Suzukı M. Tonsillectomy as part of the treatment for IgA nephropathy: cooperation between nephrologists and otolaryngologists. Clin Exp Nephrol. 2007; 11(3): 258-9.

13. Appel GB, Waldman M. The IgA nephropathy treatment dilemma. Kidney Int 2006; 69(11): 1939-44.

14. Akagi H, Kosaka M, Hattori K, Dol A, Fukushima K, Okano M, Kariya S, Nishizaki K, Sugiyama N, Shikata K, Makino H, Masuda Y. Long-term results of tonsillectomy as a treatment for IgA nephropathy. Acta Otolaryngol Suppl 2004; (555): 38-42.

15. Chen Y, Tang Z, Wang Q, Yu Y, Zeng C, Chen H, Liu ZH, LI LS. Long-term efficacy of tonsillectomy in Chinese patients with IgA nephropathy. Am J Nephrol 2007; 27(2): 170-5.

16. HоттA 0 . Tonsillectomy combined with steroid pulse therapy: a curative therapy for $\lg A$ nephropathy. Acta Otolaryngol Suppl 2004; (555): 43-8.

17. Hotta 0, Furuta T, Chiba S, Tomioka S, Taguma Y. Regression of IgA nephropathy: a repeat biopsy study. Am J Kidney Dis 2002; 39(3): 493-502.

18. Hotta 0, Miyazaki M, Furuta T, Tomioka S, Chiba $S$, Horigome I, Abe K, Taguma Y. Tonsillectomy and steroid pulse therapy significantly impact on clinical remission in patients with IgA nephropathy. Am J Kidney Dis 2001; 38(4): 736-43.

19. Sato M, Hotta O, Tomioka S, Horigome I, Chiba S, Miyazakı M, Noshiro H, TAGuma Y. Cohort study of advanced IgA nephropathy: efficacy and limitations of corticosteroids with tonsillectomy. Nephron Clin Pract 2003; 93(4): c137-45.

20. Kawasaki $Y$, Takano $K$, Suyama $K$, Isome M, SuzukI $H$, Sakuma $H$, FuJikI T, SuzUKI H, Hosoya M. Efficacy of tonsillectomy pulse therapy versus multiple-drug therapy for IgA nephropathy. Pediatr Nephrol 2006; 21(11): 1701-6.

21. Xie Y, Nishi S, Ueno M, Imai N, Sakatsume M, Narita I, Suzuki Y, Akazawa K, Shimada H, Arakawa $M$, Gesyo $F$. The efficacy of tonsillectomy on long-term renal survival in patients with IgA nephropathy. Kidney Int 2003; 63(5): 1861-7.

21. Tomioka S, Miroshi K, Tabata K, Hotta 0, Taguma $Y$. Cinical study of chronic tonsillitis with IgA 
nephropathy treated by tonsillectomy. Acta Otolaryngol Suppl 1996; 523: 175-7.

22. Sanal A, Kudoh F. Effects of tonsillectomy in children with $\operatorname{IgA}$ nephropathy, purpura nephritis, or other chronic glomerulonephritides. Acta Otolaryngol Supp/ 1996; 523: 172-4.

23. Rasche FM, Schwarz A, Keller F. Tonsillectomy does not prevent a progressive course in $\lg A$ nephropathy. Clin Nephrol 1999; 51(3): 14752.

24. Nishi S, Xie Y, Ueno M, Imai N, Suzuki Y, Iguchi S et AL. A clinicopathological study on the long-term efficacy of tonsillectomy in patients with IgA nephropathy. Acta Otolaryngol Suppl 2004; (555): 49-53.

25. Snider LA, Swedo SE. Post-streptococcal autoimmune disorders of the central nervous system. Curr Opin Neurol 2003; 16(3): 359-65.

26. Betancourt YM, JimÉnez-León JC, JimÉnezBetancourt CS, Castillo VE. Autoimmune neuropsychiatric disorders associated to infection by streptococcus in the paediatric age: PANDAS. Rev Neurol 2003; 36 Suppl 1: S95-107.

27. Kim SW, Grant JE, Kim SI, Swanson TA, Bernstein GA, Jaszcz WB, Williams KA, Schlievert PM. A possible association of recurrent streptococcal infections and acute onset of obsessivecompulsive disorder. J Neuropsychiatry Clin Neurosci 2004; 16(3): 252-60.

28. Van Toorn R, Weyers HH, Schoeman JF. Distinguishing PANDAS from Sydenham's chorea: case report and review of the literature. Eur J Paediatr Neurol 2004; 8(4): 211-6.

29. Orvidas LJ, Slattery MJ. Pediatric autoimmune neuropsychiatric disorders and streptococcal infections: role of otolaryngologist. Laryngoscope 2001; 111(9): 1515-9.

30. Heubi C, Shott SR. PANDAS: pediatric autoimmune neuropsychiatric disorders associated with streptococcal infections-an uncommon, but important indication for tonsillectomy. Int J Pediatr Otorhinolaryngol 2003; 67(8): 837-40.

31. Batuecas Caletrío A, Sánchez González F, Santa Cruz Ruiz S, Santos Goruón P, Blanco Pérez P. PANDAS Syndrome: A New Tonsillectomy
Indication? Acta Otorrinolaringol Esp 2008; 59(7): 362-3.

32. Lynch NE, Deiratany S, Webb DW, Mcmenamin JB. PANDAS (Paediatric Autoimmune Neuropsychiatric Disorder Associated with Streptococcal Infection). Ir Med J 2006; 99(5): 155.

33. Feder HM JR. Periodic fever, aphthous stomatitis, pharyngitis, adenitis: a review of a new syndrome. Curr Opin Pediatr 2000; 12 : 253-256.

34. Padeh S, Brezniak N, Zemer D, Pras E, Livneh A, Langevitz P. Periodic fever, aphthous stomatitis, pharyngitis and adenopaties syndrome: clinical characteristics and outcome. J Pediatr 1999; 135: 98-101.

35. Thomas KT, Feder HM JR, Lawton AR, Edwards KM. Periodic fever syndrome in children. $J$ Pediatr 1999; 135: 15-21.

36. Femiano F, Lanza A, Buonaiuto C, Gombos F, Cirillo N. Oral aphthous-like lesions, PFAPA syndrome: a review. J Oral Pathol Med 2008; 37(6): 319-23.

37. Pillet P, Ansoborlo S, Carrere A, Perel $Y$, GuILLARD JM. PFAPA syndrome: value of cimetidine. Arch Pediatr 2000; 7: 54-57.

38. Tasher D, Somekh E, Dalal I. PFAPA syndrome: new clinical aspects disclosed. Arch Dis Child 2006; 91(12): 981-4.

39. Renko M, Salo e, Putto-laurila a, Saxen h, Mattlla PS, Luotonen J et al. A randomized, controlled trial of tonsillectomy in periodic fever, aphthous stomatitis, pharyngitis, and adenitis syndrome. J Pediatr 2007; 151(3): 289-92.

40. Licameli G, Jefrrey J, Luz J, Jones D, Kenna M. Effect of adenotonsillectomy in PFAPA syndrome. Arch Otolaryngol Head Neck Surg 2008; 134(2): 136-40.

41. Wong KK, Finlay JC, Moxham JP. Role of Tonsillectomy in PFAPA Syndrome. Arch Otolaryngol Head Neck Surg 2008; 134(1): 16-9.

42. Galanakis E, Papadakis CE, Giannoussi E, Karatzanis AD, Bitsori M, HeLIDOnIs ES. PFAPA syndrome in children evaluated for tonsillectomy. Arch Dis Child 2002; 86(6): 434-5.

43. Berlucchi M, Meinı A, Plebani A, Bonvinı MG, Lombardi D, Nicolal P. Update on treatment of Marshall's syndrome (PFAPA syndrome): 
report of five cases with review of the literature. Ann Otol Rhinol Laryngol. 2003 Apr; 112(4): 365-9.

44. Dahn KA, Glode MP, Chan KH. Periodic fever and pharyngitis in young children: a new disease for the otolaryngologist? Arch Otolaryngol Head Neck Surg. 2000; 126(9): 1146-9.

45. Parikh SR, Reiter ER, Kenna MA, Roberson D. Utility of tonsillectomy in 2 patients with the syndrome of periodic fever, aphthous stomatitis, pharyngitis, and cervical adenitis. Arch Otolaryngol Head Neck Surg 2003; 129(6): 670-3.

46. Sakiyama $H$, Kobayashi S, Dianzani $U$, Ogiuchi $H$, Kawashima M, Uchiyama T, Yagl J. Possible involvement of $T$ cell co-stimulation in pustulosis palmaris et plantaris via the induction of inducible co-stimulator in chronic focal infections. Dermatol Sci 2008; 50(3): 197-207.

47. Gudjonsson JE, Thorarinsson AM, Sigurgeirsson B, Kristinsson KG, Valdimarsson H. Streptococcal throat infections and exacerbation of chronic plaque psoriasis: a prospective study. $\mathrm{Br} \mathrm{J}$ Dermatol 2003; 149(3): 530-4.

48. McMillin BD, Maddern BR, Graham WR. A role for tonsillectomy in the treatment of psoriasis? Ear Nose Throat J 1999; 78(3): 155-8.

49. Nyfors A, Rasmussen Pa, Lemholt K, Eriksen B. Improvement of refractory psoriasis vulgaris after tonsillectomy. Dermatologica 1975; 151 : 216-222.

50. Hone SW, Donnelly MJ, Powell F, Blayney AW. Clearance of recalcitrant psoriasis after tonsillectomy. Clin Otolaryngol 1996; 21: 546547.

51. Unver S, Kubilay U, Sezen Os, Coskuner T. Investigation of Helicobacter pylori colonization in adenotonsillectomy specimens by means of the CLO test. Laryngoscope 2001; 111(12): 21836.

52. Vestergaard $H$, Westergaard $T$, Wohlfahrt J, Pipper C, Melbye M. Association between intussusception and tonsil disease in childhood. Epidemiology 2008; 19(1): 71-4.

53. OvChinsky A, SCHULman S, Rosenfeld RM. Adenotonsillectomy as a treatment option for poststreptococcal uveitis. Laryngoscope 2002; 112(4): 658-60.

54. Mucha K, Foroncewicz B, Niemczyk K, ZiarkiewiczWróblewska B, Stanisawek-Sut 0, Zieniewicz K, Krawczyk M, Paczek L. Tonsil enlargement after liver transplantation in adults-reason enough for tonsillectomy? Two cases of tonsillar posttransplantation lymphoproliferative disease. Liver Transp/ 2007; 13(6): 918-23.

55. Nouwen J, Smets F, Rombaux P, Hamoir M, Sokal EM. Acute tonsillitis as the first manifestation of post-transplant lymphoproliferative disorder. Ann Otol Rhinol Laryngol 2002; 111(2): 165-8.

Dirección: Dr. Daniel Muñoz S.

Av. Independencia 939, Independencia, Santiago

E mail: daniel.uchile@gmail.com 